Lapurdum

Euskal ikerketen aldizkaria | Revue d'études basques |

Revista de estudios vascos | Basque studies review

$4 \mid 1999$

Numéro IV

\title{
Les noms de montagnes du Pays Basque
}

Michel Morvan

\section{OpenEdition \\ Journals}

Édition électronique

URL : http://journals.openedition.org/lapurdum/1551

DOI : 10.4000/lapurdum. 1551

ISSN : 1965-0655

Éditeur

IKER

Édition imprimée

Date de publication : 1 octobre 1999

Pagination : 167-190

ISBN : 2-84127-156-0

ISSN : $1273-3830$

Référence électronique

Michel Morvan, «Les noms de montagnes du Pays Basque », Lapurdum [En ligne], 4| 1999, mis en ligne le 01 avril 2010, consulté le 21 décembre 2020. URL : http://journals.openedition.org/lapurdum/ 1551 ; DOI : https://doi.org/10.4000/lapurdum.1551 


\title{
Michel MORVAN
}

\section{LES NOMS DE MONTAGNES DU PAYS BASQUE}

\author{
A Jean Haritschelhar, \\ à l'humaniste.
}

Abréviations : comm. =commune(s), IGN=Institut Géographique National, $\mathrm{m} .=$ mètres, suff. $=$ suffixe, var. $=$ variante.

Abarakoharria $581 \mathrm{~m}$ comm. de Bidarray et d'Ossès.

On pense tout de suite à un dérivé de abar "branche". On aura donc ici probablement un composé abarra avec *abarr $+a$ (ce - $a$ final pouvant être une contraction du suff, -aga "lieu" plutôt que l'article basque postposé) + ko génitif + harria "la pierre, la roche". Soit "la roche du lieu des branchages".

Abarratia 342 m comm. d'Isturits.

Comme précédemment on pense à $a b a r$ "branchage" ou à un de ses dérivés comme abari "chêne vert". L'élément - $t i$ peut soit être di / $t i$ "lieu", soit simplement -te avec fermeture de la voyelle en $i$ devant l'article, auquel cas il faut analyser *abarr-ate suivi de l'article. L'élément ate est soit ate "col, port, passage", soit simplement la var. de eta "lieu" bien attestée en toponymie basque (cf. Garate, Haltzate, etc.). On retiendra cette dernière solution (avec haltz "aulne" par ex. il ne peut s'agir d'un col de montagne élevé étant donné que cet arbre pousse plutôt dans les plaines au bord des cours d'eau).

Abératcouné $391 \mathrm{~m}$ comm. d'Ossès.

Nom composé de abere ou aberats "bétail, richesse" et de une "lieu" : du nom de maison Aberatxenea. Doit être écrit Aberatxune.

Abozé 586 m comm. d'Espelette.

Bien que situé en dehors de la Soule, il n'est pas interdit d'envisager pour cet oronyme un nom terminé par le mot aujourd'hui uniquement souletin botxe, boxi "rocher, escarpement". Le premier élément demeure également obscur. On notera la ressemblance avec l'oronyme Zabozé.

Abrakou 885 m comm. des Aldudes.

Nous avons de nouveau affaire à $a b a r$ "branchage" + suff. - ko. La contraction de $a b a r(r) a$ en abra n'a rien de surprenant surtout dans les dialectes orientaux.

Achaldaxa $170 \mathrm{~m}$ comm. de Charritte-de-Bas.

Nom composé de aitz "pierre, roche" et de alda "côte, pente"?

Achistoy $1230 \mathrm{~m}$, comm. de Banca.

Il s'agit ici sans doute d'une forme complexe déformée à partir de aitz "pierre" ou mieux haitz "arbre, chêne" suivi du suff. collectif doi/toi "lieu de, 
ensemble de". Une contraction de axeri "renard" est très peu vraisemblable en oronymie basque.

Achourterrigagna $1660 \mathrm{~m}$, comm. de Larrau.

Un composé de aitz "pierre" + ur "eau" ("eau de roche") est inconnu en toponymie basque. Il s'agit sans doute de axuri "agneau" suivi de herri "pays" (le - $t$ n'étant qu'une liaison semble-t-il) ou (h) arri "pierre, roche" et de gain "hauteur". Il existe en Soule une maison nommée Axurtidegi sur la carte IGN, mais sa forme ancienne est Axurbidegi au Moyen Âge, avec sans aucun doute bide "chemin" comme élément central (crête du chemin des agneaux).

Adarza $1250 \mathrm{~m}$, comm. de Banca.

Il n'y a rien d'étonnant à trouver le terme adar "branche, corne" en oronymie. C'est un mot que l'on peut relever dans d'autres langues que le basque, par exemple en oronymie française avec Banne "corne", vieux terme gaulois ou prégaulois, ou encore en oronymie germanique avec Horn (cf. le Matterhorn en Suisse). On aura donc ici adar "corne" suivi du suff, -tza.

Aguerria $421 \mathrm{~m}$ comm. d'Ainharp.

L'étymologie de ce nom est évidente, c'est le cas de le dire, puisqu'on a ici agerri "visible, en vue" ou mieux agerre "id." avec fermeture du $e$ en $i$ devant l'article basque (agerrea $>$ agerria). Soit "la (hauteur) visible, en vue".

Ahargo $609 \mathrm{~m}$, comm. de Barcus.

On pense tout de suite à ahari "mouton", mais rien ne prouve que ce soit la bonne interprétation. La chute d'une ancienne consonne initiale est tout à fait possible, par ex. on pourrait avoir ici un ancien *lahargo, avec lahar "ronce" comme premier élément. Le $l$ - initial ayant ensuite été pris à tort pour l'article roman.

Ahaxamendy $330 \mathrm{~m}$, comm. de Ahaxe-Alciette-Bascassan.

Bien évidemment il faut mettre en relation directe le nom de cette hauteur modeste avec celui du village d'Ahaxe, soit "montagne d'Ahaxe". Rappelons ici qu'en basque, le terme mendi "montagne" peut s'appliquer à toute hauteur, même fort peu élıvée.

Ahiga $300 \mathrm{~m}$, comm. de Lohitzun-Oyhercq.

Nom obscur. On peut proposer un ancien Ihiaga "lieu de joncs". On remarquera sur la même commine la présence d'un oronyme Aldiga.

Ahismeaka $773 \mathrm{~m}$, comm. de Lasse.

D'après P. Lhande, mehaka pourrait avoir le sens de "éminence", ce qui est impossible. Le terme final est bien sûr mehaka "défilé, passage étroî". Quant au premier élément Ahis-, il faut sans doute y voir aitz "pierre, roche" dont l'évolution aitz $>$ ahaiz $>$ ahiz est tout à fait régulière. On a donc ici "défilé rocheux".

Ahuntzbide 1394 m, comm. de Lécumberry.

Écrit Ahunsbide chez P. Raymond, voici un nom sans problème, composé de ahuntz "chèvre" et de bide "chemin". L'IGN écrit "crête d'Ahuntzbide".

Ahounsbiscardéguy $128 \mathrm{~m}$, comm. d'Ayherre.

L'étymologie de cet oronyme ne pose pas de problème : ahuntz "chèvre", bizkar "dos, crête", egi "bord, crête, lieu" avec dentale - $d$ - de liaison.

Ainhiza mendy $349 \mathrm{~m}$, comm d'Ainhice-Mongelos. 
C'est évidemment un nom en relation avec le village, soit la "montagne d'Ainhice".

Aintziaga $905 \mathrm{~m}$, comm. de Saint-Étienne-de-Baïgorry.

Écrit Ainciague chez P. Raymond, cet oronyme se décompose facilement en aintzi "marécage, lieu marécageux ou humide" + aga "lieu".

Airamendi $362 \mathrm{~m}$, comm. d'Irissarry et Iholdy.

Sans doute faut-il voir ici simplement un composé de aire "air" + mendi "hauteur", soit le "mont aéré" ou encore "mont d'où l'on s'envole". Une autre solution consisterait à rapprocher Aira du nom de la forêt d'Hayra.

Akerharri $883 \mathrm{~m}$, comm. de Lécumberry. bouc".

Nom composé de aker "bouc" et de harri "pierre, roche". Soit "roche du

Albista $242 \mathrm{~m}$, comm. de Lohitzun-Oyhercq.

Nom signifiant sans doute "lieu de graminées", de albitz "graminée, foin" et eta "lieu".

Aldiga $308 \mathrm{~m}$, comm. de Lohitzun-Oyhercq.

On peut envisager un composé de alde "côte, pente". La suite peut représenter un mont Ihiga (de ihi-aga "lieu de joncs"), soit "côte, versant du mont Ihiga". Voir aussi Ahiga.

Altxanga $625 \mathrm{~m}$, comm. de Sare.

Très probablement (h)altz "aulne", peut-être *altz-un-aga "lieu des aulnes".

Alupigna $1395 \mathrm{~m}$, comm. de Larrau.

Écrit Alupeña chez P. Raymond. Oronyme composé de alu "con, sexe féminin" et de peña "roche". Le sens alu "con, sexe féminin" est bien confirmé par d'autres micro-oronymes comme Aluipurdi "cul du con", aussi curieux que cela puisse paraître. Légende? Réfection analogique et anthropomorphique?

Ametzlepo $687 \mathrm{~m}$, comm. des Aldudes.

Étymologie sans problème, de ametz "chêne tauzin" et lepo "col".

Antchola $1119 \mathrm{~m}$, comm. de Banca.

Sans doute du nom propre Antso suivi de ola "cabane", bien qu'on ne puisse écarter tout à fait antxu "mouton" ou même aintzi "marécage". Le village aragonais d'Ansó n'est pas très loin. Cabane d'Antxo ou cabane des moutons?

Antzaramendi $462 \mathrm{~m}$, comm. d'Ossès.

Une explication littérale donnerait "montagne de l'oie"(antzara "oie"). Mais il s'agit peut-être d'une étymologie populaire, de même que l'on trouve des Montes de Oca du côté basque espagnol. D'autres oronymes ont pour premier élément antz-comme par ex. Antzeta.

Antzeta $433 \mathrm{~m}$, comm. de Jaxu.

Oronyme composé d'un élément obscur antz qui pourrait bien être "mouton" ou aintzi "lieu humide, marécage" et du suff. -eta.

Apolotzegagna $720 \mathrm{~m}$, comm. de Haux.

Écrit Apholotxé chez P. Raymond. Déformation probable d'un ancien apal-aitz "rocher du bas" mal transcrit ou phonétiquement altéré, suivi de gain "hauteur". Apalatzeaga existe comme nom de maison. 
Arambeaux $498 \mathrm{~m}$, comm. de Chéraute.

Nom romanisé composé à l'origine de aran "vallée" et beltz "noire".

Aranohéguy $1293 \mathrm{~m}$, comm. de Lecumberry.

Étymologie sans difficulté, de arrano "aigle" et hegi "crête".

Arbildura 292 m, comm. de Saint-Martin-d'Arberoue.

Il faut envisager ici une déformation de arbel "roche noire, ardoise" suivi de gora "hauteur" plutôt qu'un composé de harri-bildura "formation de pierres". On voit bien d'ailleurs le lien "ntre l'oronyme et le nom du village qui comporte Arberoue de même origine (arbel-), soit "hauteur d'Arberoue", l'Arberoue étant également une rivière.

Arbosse $541 \mathrm{~m}$, comm. de Uhart-Cize.

Deux hypothèses sont possibles pour expliquer cet oronyme. Ou bien il s'agit de $a r$ - "pierre, roche" + be "sous" suivi du suff. basco-aquitain -os, ou bien il s'agit d'un ancien *narb- "souche", ayant perdu le $n$ - initial, avec le même suff. -os (cp. avec le nom de lieu Arbonne, ancien Narbona).

Archilako cascoa $400 \mathrm{~m}$, comm. de Iholdy et Lantabat.

C'est sans doute un ancien arxilagako kaskoa "sommet du lieu de la pierre trouée"(arri-xilo-aga-ko), avec réduction de aga à $a$.

Arguimonho $548 \mathrm{~m}$, comm. de Saint-Just-Ibarre.

Nom composé de argi "clair" et de monho "colline".

Arradoy $660 \mathrm{~m}$, comm. d'Ispoure.

La montagne qui domine Saint-Jean-Pied-de-Port, bien abîmée sur ses flancs au demeurant par le déboisement, a un nom composé de arra- et du suff. -doi bien connu. Soit "ensemble de...". Reste à trouver le sens de arra-. Il y a de fortes chances pour qu'il s'agisse d'un ancien arrai "ćpineux", que l'on retrouve dans i $\varepsilon$ nom du village de Bidarray par ex. On retiendra donc "lieu, ensemble des épineux".

Arrolakoharria 1060 m, comm. d'Anhaux, Saint-Étienne-de-Baïgorry, Banca. Oronyme composé de arrola "ravin" + ko + harri, soit "la roche du ravin".

Arthaburu $1156 \mathrm{~m}$, comm. de Lecumberry.

Étymologie sans problème, de artha "chêne vert" et buru "sommet".

Arthanolatzégagnia 1379 m, comm. de Larrau.

A première vue, on aurait ici artha "chêne vert" et ola "cabane", mais c'est peut-être trompeur, car le - $n$ demeure inexpliqué (arthanolatze). Autrement dit il faudrait analyser plutôt ce nom en arthano (?) + latz qui pourrait représenter lats "cours d'eau" (?).

Arthé $1010 \mathrm{~m}$, comm. d'Esterençuby.

Cet oronyme peut être soit artha "chêne vert", soit un ancien *arte-mendi "hauteur intermédiaire", soit encore un composé de arri "pierre, roche" + te.

Artikulugagna $1582 \mathrm{~m}$, comm. de Larrau.

Ce curieux nom a bien peu de chances de comporter le mot artikulu "article" dont on ne voit vraiment pas ce qu'il viendrait faire là. La finale fait penser à celle du site d'Urkulu, mais cela ne nous avance guère. Il convient sans doute d'analyser ce nom à l'aide d'arta "chêne vert" et de okulu "lieu" (emprunt au lat. loculum) + gain 
Artzaïnharria $971 \mathrm{~m}$, comm. d'Anhaux.

Aucun problème. C'est "la roche du berger ou des bergers", artzain-harria. Écrit Arçainharria chez P. Raymond.

Artzamendi $926 \mathrm{~m}$, comm. d'Itxassou.

Oronyme complexe. Écrit Harţ̧amendy chez P. Raymond. Le - $a$ final de artza peut représenter un ancien -aga ou un ancien $e$ dans hartze "lieu pierreux" qui devient hartza dans les noms composés. Bien sûr il y a aussi artza "ours", mais le problème se complique avec l'existence d'un autre oronyme Haltzamendi dont le sens n'est pas clair non plus, car le nom d'arbre haltz "aulne" convient mal en altitude.

Ascune $860 \mathrm{~m}$, comm. d'Aussurucq.

Ce pic d'Ascune s'analyse aisément en aitz-gune "lieu de roche".

Askopé $384 \mathrm{~m}$, comm. de Biriatou.

Sans doute avons nous ici un simple aitz-ko-pe "sous la roche, en bas de la roche", avec peut-être un ko diminutif.

Astate 1022 m, comm. de Saint-Étienne-de-Baïgorry.

Écrit Astaté chez P. Raymond. Sans doute de aitz "roche" + ate "col, passage".

Atchapuru 409 m, comm. de Saint-Esteben.

Oronyme dérivé de aitz "pierre, roche" avec un - a final qui nous ramène sans doute au suff. -aga contracté, car la forme normale simple est aitzpuru. Donc "sommet, extrémité du lieu de roche".

Athekaleun $813 \mathrm{~m}$, comm, de Sare.

Étymologie sans problème. De atheka "col, passage" et leun "doux, lisse" (ici avec le sens de "facile à franchir").

Atxuria $756 \mathrm{~m}$, comm. de Sare.

Le terme axuri "agneau" est peu probable ici. La dentale nous invite à comprendre aitz-xuria "la roche blanche".

Aurkhilarré $451 \mathrm{~m}$, comm. d'Ossès.

Nom composé de aurkhi "devant" et de larre "lande".

Azaléguy $583 \mathrm{~m}$, comm. de Saint-Just-Ibarre.

Écrit Açaléguy chez P. Raymond. La difficulté réside ici dans l'explication à donner du $-l$ - de azal qui peut ne pas appartenir à ce premier élément, mais être parfois une liaison comme dans Behorleguy. Toutefois aza seul ne veut rien dire. Une explication à partir de azal "croûte" étant peu probable, il faut envisager aitz "pierre, roche" suivi de ala "pâturage". Soit "crête du pâturage rocheux".

Babatze $404 \mathrm{~m}$, comm. de Bunus.

Étant donné la faible hauteur de cet oronyme, on ne peut exclure baba "fève", avec une culture de fèves sur le versant.

Bakuitze $404 \mathrm{~m}$, comm. d'Irissarry.

Il s'agit ici probablement de bakoitz "isolé".

Baygoura $818 \mathrm{~m}$, comm. d'Ossès.

Voilà un oronyme qui a déjà fait couler beaucoup d'encre. On a tout d'abord rapproché ce nom de celui de Baigorry "rivière rouge". Il est vrai que c'était très tentant, d'autant plus que l'on a Baiguer parmi les formes romanisées de 
Baigorry. Mais il y a un élément phonétique rédhibitoire qui s'oppose à une explication du second élément par gorri "rouge". En effet la prononciation de Baigura montre toujours un $r$ simple et non un $r r$ fort. Il faut donc rattacher gura à gora "hauteur" ou à guren "limite".

Begousse $767 \mathrm{~m}$, comm. de Montory.

Écrit Bégousse chez P. Raymond. A Montory, ce nom de montagne a de fortes chances d'être gascon ou d'appartenir au substrat pré-gascon avec suff. aquitain -os. On pourra comparer avec des noms de lieux gascons comme La Bégoussère, écart de la commune de Sainte-Suzanne (Béarn), Les Bégossères en 1779.

Béharria $880 \mathrm{~m}$, comm. de Lasse et Anhaux.

Sans doute doit-on penser ici à un anthropomorphisme beharri "oreille" (roche en forme d'oreille).

Behorléguy $1265 \mathrm{~m}$, comm. de Béhorléguy.

Le nom de ce pic est un composé de behor "jument" et de egi "crête, bord, lieu" avec un $-l$ - de liaison fréquent en toponymie ou oronymie basque. Cette "crête de la jument" est confirmée par d'autres oronymes, même en dehors du domaine basque, cf. l'oronyme La Jeugue en Béarn.

Behorsaro $500 \mathrm{~m}$, comm. d'Ossès.

Nom composé de behor "jument" et de saro "lieu de rassemblement des troupeaux".

Beillurti $1114 \mathrm{~m}$, comm. d'Arnéguy.

Écrit Beillurte chez. P. Raymond. La forme donnée par Raymond est sans doute plus proche de l'origine. Le $e$ s'est ensuite fermé en $i$. Quant à l'analyse, elle est assez complexe. On pourrait penser à behi "vache", mais cela n'explique guère le reste de l'oronyme. Il semble bien que l'on soit en présence d'un nom contenant le terme elur "neige", peut-être précédé de be "en bas", soit "le bas du lieu où la neige persiste".

Belchou 1129 m, comm. de Saint-Just-Ibarre.

Oronyme dérivé de beltz "noir, sombre", avec suff. -o qui se ferme en $u$.

Belhay $1771 \mathrm{~m}$, comm. de Larrau.

Écrit Belay hez P. Raymond. Dérivé de bel- "noir" ou de belar "herbe". Soit "pâturage". Il existait une maison noble Belay à Biarritz.

Belhygagne $1072 \mathrm{~m}$, comm. d'Alçay-Alçabéhéty-Sunharette.

Écrit Belhy chez P, Raymond. Ce pourrait être la "hauteur des corbeaux" (bele "corbeau").

Beloscare 1173 m, comm. de Lacarry-Charritte de Haut.

Écrit Beloscar chez P. Raymond. Ce nom est bien problématique. On le retrouve également comme nom de maison médiévale en Soule.

Berhantchu $574 \mathrm{~m}$, comm. de Musculdy.

Nom obscur dérivé de bel "noir" ou peut-être de behere "en bas" + antxu "mouton".

Betzulagagna $1590 \mathrm{~m}$, comm. de Larrau.

Écrit Betçula chez P. Raymond. Il pourrait s'agir ici d'un beltz-ola "cabane noire" déformé (?) + gain "hauteur". 
Bimbalèta 1758 m, comm. de Sainte-Engrâce.

Écrit Bimbalette chez P. Raymond. Oronyme dont le sens est "clochette", allusion à l'estive bien sûr.

Bizkarzé $1656 \mathrm{~m}$, comm. de Larrau.

Étymologie sans problème, de bizkar "dos, crête" avec suff. -tz. On trouve un nom identique en Béarn, sur les communes de Bedous et Sarrance (Biscarce).

Bizkarzun 185 m comm. de Saint-Pée-sur-Nivelle et Ascain.

Oronyme composé de bizkartz (voir le précédent) + un "lieu".

Bizkayluze $671 \mathrm{~m}$, comm. d'Espelette et d'Itxassou.

Oronyme composé de bizkai "crête" et luze "longue".

Bohorcortia $1214 \mathrm{~m}$, comm. de Larrau.

Oronyme composé de bohor "jument" (forme normale en Soule pour behor) et de gorte "aire, cour, parc, bergerie". Soit "le parc aux juments".

Bosmendiette $1000 \mathrm{~m}$, comm. de Larrau.

Écrit Bostmendy chez P. Raymond. Nom bien connu grâce à la chanson de Berterretche datant du XVe siècle, mais recueillie seulement au XIX". Soit "lieu des cinq montagnes".

Buhuzé $244 \mathrm{~m}$, comm. d'Armendarits.

Oronyme de sens incertain. Peut-être de bun(u)- "hauteur, colline"(cf. le toponyme Bunus) $+t z+e$.

Burdincurutcheta $1092 \mathrm{~m}$, comm. d'Urepel et Banca.

Nom composé de burdin "fer" + kurutze "croix" + eta "lieu". Soit "lieu de la croix de fer".

Buztanzelhay $1029 \mathrm{~m}$, comm. de Saint-Étienne-de-Baïgorry.

A première vue, c'est "plateau de l'extrémité", avec buztan "queue, extrémité", mais buztan peut aussi être une var. de buztin "argile", qui semble préférable ici.

Chamboroy $503 \mathrm{~m}$, comm. de Saint-Étienne-de-Baïgorry.

Écrit Chunboroy chez P. Raymond. Curieux nom qui doit être bien déformé. On peut envisager quelque chose comme Altzumberro "taillis des aulnes" que l'on a par ex. dans le nom de village Sumberraute. On trouve aussi un toponyme Chamberro sur la commune d'Ossès dit "Chamborro".

Chardéca $1555 \mathrm{~m}$, comm. de Larrau.

Écrit Chardaca chez P. Raymond. Étymologie sans problème, de xardeka "fourche". Pic de la fourche.

Chardékagagna $1881 \mathrm{~m}$, comm. de Larrau.

Même nom que le précédent.

Cherrapo $299 \mathrm{~m}$, comm. de Çaro.

Nom obscur. Zerrapo est "serrure".

Cherrenda $218 \mathrm{~m}$, comm. de Mendionde.

Nom pouvant représenter zerrenda "rangée" au sens de "bande de terrain".

Chouri Punta 1621 m, comm. de Sainte-Engrâce.

Étymologie simple : pointe blanche. On notera l'adjectif antéposé, inhabituel en basque moderne. 
Coheguy 785 m, comm. de Lécumberry.

Nom déformé supposant la perte d'un premier élément + egi "crête".

Coscohandy $481 \mathrm{~m}$, comm. de Uhart-Cize.

Nom composé de kosko "sommet" pour kasko et handi "grand".

Cotroil 355 m, comm. d'Ossès.

Nom dérivé de kotor "côte, pente".

Curutchamendy $290 \mathrm{~m}$, comm. de Uhart-Cize.

Nom sans problème, composé de kurutze "croix" et de mendi "montagne, hauteur". Le - $a$ - peut représenter soit une évolution $-e>-a$, soit un ancien -aga.

Curutchéberry $513 \mathrm{~m}$, comm. de Larceveau-Arros-Cibits.

Nom composé de kurutze "croix" et de berri "nouvelle".

Curutchehegui $486 \mathrm{~m}$, comm. de Lantabat.

Nom composé de kurutze "croix" et de hegi "crête".

Daraturugagne 494 m, comm. de Pagolle.

Ecit Darraturu chez P. Raymond. Le terme basque daraturu "tarière" ne convient pas en oronymie. Il s'agit certainement d'une déformation d'un autre mot, peut-être bien de larre "lande".

Droundak $1628 \mathrm{~m}$, comm. de Sainte-Engrâce.

Le basque drunda "mèche" ne convient pas. Il faut peut-être envisager un terme expressif (grondement d'orage?). On notera que Dronde existe comme nom de personne en Soule, et nom de maison.

Egurmendy 986 m, comm. de Béhorléguy.

Nom composé de egur "bois de chauffage" et de mendi "montagne".

Eheta 637 m, comm. de Gamarthe.

Nom obscur. On peut cependant envisager sérieusement un ancien Leheta "lieu de pins" (de leher "pin") ayant perdu son initiale.

Ekhi Altia 633 m, comm. de Sainte-Engrâce.

Nom composé de eki "soleil" et de alte "côté".

Elaudy $700 \mathrm{~m}$, comm. de Musculdy.

On pense à elur "neige", mais cela n'explique pas le segment -au-. Autre possibilité, elhorri-di "lieu d'épineux", peut-être réduit à elhoi-di et devenu elodi, puis elaudi avec-au-sous l'influence du français.

Elhorriko kaskoa $983 \mathrm{~m}$, comm. de Saint-Étienne-de-Baïgorry.

Nom sans problème : de elhorri "épine" et kasko "sommet". Soit "sommet des épineux".

Elhuroso lepoua $1650 \mathrm{~m}$, comm. de Larrau.

On a sans doute ici un nom composé de elhur "neige" avec lepo "col". La finale -so représente sans doute un ancien suff. - $t s u$ "qui abonde en".

Elissamendia $313 \mathrm{~m}$, comm. de Moncayolle-Larrory-Mendibieu.

C'est simplement "la hauteur de l'église", soit parce que l'église y était située, soit parce qu'il s'agit d'une terre (lande) appartenant à l'église. 
Elsarré $1153 \mathrm{~m}$, comm. d'Aussurucq.

Écrit Elçarré chez P. Raymond. Nom composé probablement de ele "pâturage" et de zahar "ancien".

Eltzarruzé $421 \mathrm{~m}$ comm. de Saint-Martin-d'Arberoue.

Le basque eltzaur "noyer" ne convient pas, car dans la région concernée on dit intzaur. De plus il faut expliquer le $u$ final. On pourrait envisager un nom similaire au précédent Elsarré. La faible hauteur permet aussi de penser à haltz "aulne" + arroz ou haltz + arr + auz.

Endiotzia $775 \mathrm{~m}$, comm. de Larrau.

Nom obscur. Peut-être une déformation de andu "souche" avec suff. -oz. Cf. aussi le nom Andoz connu en Soule.

Ereby 583 m, comm. d'Espelette.

Écrit Erreby chez P. Raymond. La graphie de Raymond avec $r r$ semble préférable, ce qui permet d'analyser cet oronyme en erro "souche" et $i b i$ "gué", sans doute apparenté à Errobi nom basque de la Nive, dont il n'est sans doute qu'un simple doublet.

Erintsu $422 \mathrm{~m}$, comm. d'Urrugne.

Nom obscur. Le suff. d'abondance - $t$ su permettrait de rétablir un $l$ - initial qui nous ramènerait à lerintsu "où les pins abondent", de ler, leher "pin".

Errayzé gagna $1451 \mathrm{~m}$, comm. de Larrau.

On a sans doute encore affaire ici à erro "souche", en composition avec aitz "pierre, roche". Soit "hauteur de la pierre aux souches".

Erréta 702 m, comm. d'Estérençuby.

Le premier élément semble être une réduction de errege "roi" suivie du suff. -eta "lieu". Soit "terre du domaine royal".

Errola 907 m, comm. de Banca et Urepel.

On a sans aucun doute de nouveau erro "souche" avec ola "cabane".

Errozate $1345 \mathrm{~m}$, comm. de Lécumberry et Estérençuby.

Nom composé sans doute de erro "souche" + l'oronyme au(t)z+ suff. ate (ou bien ate "passage, col").

Escouareguibelgagna $629 \mathrm{~m}$, comm. de Camou-Cihigue.

Étymologie complexe. On peut analyser la première partie de ce nom soit avec aitz "pierre" + ku + aurre "devant", soit à l'aide d'une déformation de ezkur "gland, chêne" suivi de gibel "arrière" et gain "hauteur".

Eskantolha $1511 \mathrm{~m}$, comm. de Sainte-Engrâce.

Écrit Escantola chez P. Raymond. On doit envisager ici ezkanda "chênaie" et ola "cabane" avec passage de nd à nt en zone souletine. Soit "cabane de la chênaie".

Esnaur 273 m, comm. d'Ascain.

Nom composé de esne "lait" et de aur "devant". Ces noms contenant esne "lait" apparaissent en zone de pâturage. Cf. le toponyme Esnazu dans la Vallée des Aldudes.

Esteinumendi $346 \mathrm{~m}$, comm. de Bidarray.

Esteinu est un nom de maison cité au XVII" siècle : "Mont d'Esteinu" 
Etchecortia 1204 m, comm. d'Aussurucq.

Cet oronyme qui signifie "cour ou parc de la maison" a dû prendre son nom d'un lieu-dit voisin ayant servi de parc à bestiaux pour les maisons du lieu.

Eyharza 868 m, comm. des Aldudes.

Nom composé de eihar "sec" et du suff. collectif -tza. Soit "lieu sec ou désséché". On comparera avec le toponyme Eyharce, hameau de la commune d'Ossès, nom de plusieurs maisons, etc.

Eyheramendy $500 \mathrm{~m}$, comm. d'Aincille et d'Estérençuby.

Nom composé de eyhera "moulin" et de mendi "montagne". Soit "montagne du moulin".

Ezpatagagna $1535 \mathrm{~m}$, comm. de Larrau.

Nom sans problème, de ezpata "épée" et gain "hauteur", sommet de l'épée, c'est-à-dire sans doute "en forme d'épée".

Faague $552 \mathrm{~m}$, comm. de Sare.

Écrit Fague chez P. raymond. De toute évidence il s'agit de fago, var. de bago "hêtre", faga en composition, réduit par haplologie à fa- devant le suff. de lieu aga $\left({ }^{*}\right.$ fago-aga $>$ faga-aga $>$ faaga $)$. C'est un procédé morphologique régulier.

Faalegi $496 \mathrm{~m}$, comm. de Biriatou.

Comme pour le Faague précédent de Sare, on a faga "hêtre" $+-l$ - de liaison + egi "crête, bord".

Gainzale $531 \mathrm{~m}$, comm. de Bunus.

Cet oronyme curieux à première vue s'explique sans doute par gain "hauteur" + zabal "plateau, plat" réduit à zaal $>z a l$ avec $-e$ final postiche.

Gakoéta 579 m, comm. de Bidarray.

On ne peut que songer aux célèbres gorges de Kakoueta de la Soule. En outre il y a un autre toponyme Kakoueta sur la commune de Musculdy. Bien sûr il faut partir de gako "crochet", soit "lieu en forme de crochet, lieu d"aspect tortueux". La proximité du gascon-béarnais et son influence romane fait passer gako à kako en Soule.

Galarreguy 247 m, comm. de Saint-Martin-d'Arberoue.

Une seule explication possible pour cet oronyme modeste: galar "bois mort, sec" et egi "crête, bord".

Garatégaina $200 \mathrm{~m}$, comm. d'Ascarat.

On a ici un garate nom de maison signifiant "lieu élevé", ou "passage élevé", cf Garai "élevê".

Garhondoko punta 960 m, comm. de Sainte-Engrâce.

Littéralement garhondo signifie "cou, nuque, arrière du crâne" en basque, d'où on pourrait tirer "col" de montagne, mais en fait gar n'est pas ici "crâne", mais "roche" (de même étymologie d'ailleurs). C'est donc la "pointe du col rocheux".

Garralda $470 \mathrm{~m}$, comm. de Hélette et de Saint-Esteben.

Oronyme simple composé de gar "roche" et de alda "côte, pente". Soit "la pente rocheuse". 
Gastelariko botchia $894 \mathrm{~m}$, comm. de Larrau.

Nom souletin composé de gaztelu "château" au sens de "défense proto-historique" + harri "pierre, roche" + ko génitif + botxe "rocher escarpé, précipice", ce dernier terme étant typiquement souletin.

Gastellaya $479 \mathrm{~m}$, comm. de Chéraute.

De gaztelu "forteresse" bien sûr, avec ai "versant" comme second élément de composé. Des vestiges sont d'ailleurs signalés sur la carte IGN. Soit "le versant du château fort".

Gastelu $629 \mathrm{~m}$, comm. de Lécumberry.

Aucun problème. Le terme ne désigne toutefois pas nécessairement un château fort réel. Il peut aussi indiquer un site de défense proto-historique.

Gastélugagna $658 \mathrm{~m}$, comm. de Sainte-Engrâce.

Aucun problème. La "hauteur du château fort".

Gastelusare $416 \mathrm{~m}$, comm. de Lantabat et de Larceveau-Cibits-Arros.

Écrit Gastelluçar chez P. Raymond. C'est le "vieux château fort" bien sûr, avec zahar "vieux" réduit à zar.

Gatarre $750 \mathrm{~m}$, comm. d'Estérençuby.

Nom formé d"une très vieille racine *gat- qui a pu signifier "escarpé". On la trouve aussi en gascon dans l'ancien nom de Betharram qui était Gatarram. De même la finale-arre existe aussi en gascon où on la trouve même seule comme oronyme ou toponyme (Arre). Il s'agit peut-être bien de la fameuse base pré-celtique *arr- "pierre, roche", apparentée au basque harri "id".

Gathuly 684 m, comm. de Banca et Saint-Étienne-de-Baïgorry.

Ce nom est très probablement gatulu "bol", soit hauteur ou montagne en forme de bol.

Gaztarrigagna 1732 m, comm. de Larrau.

Oronyme qui pourrait s'analyser à partir de gatz "sel" ou gazta "fromage".

Gaztélia 1345 m, comm. d'Alçay-Alçabéhéty-Sunharette.

Aucun problème. C'est une "forteresse" de site proto-historique, Le - $u$ final de gaztelu se ferme en $-i$ devant l'article.

Gazteluzahar $472 \mathrm{~m}$, comm. de Lantabat.

Nom sans problème signifiant "vieille forteresse".

Gnabarignégagné $1066 \mathrm{~m}$, comm. de Sainte-Engrâce.

Ce curieux nom, typique des déformations que l'on peut rencontrer dans le domaine du souletin, semble être composé soit de inhabar, soit de naba + harri + gain

Gorospil 656 m, comm. d'Ainhoa.

Écrit Gorospila chez P. Raymond. Nom composé de goro-tz "houx" et bil "sommet arrondi".

Goyburu 1203 m, comm. de Sainte-Engrâce.

Nom composé de goi "haut" et de buru "tête, extrémité", soit la "pointe haute".

Haitzeder 134 m, comm. d'Hasparren.

Nom composé de haitz "pierre, roche" et de eder "belle". 
Handiamendi $642 \mathrm{~m}$, comm. d'Aincille et d'Estérençuby.

Le nom semblerait clair, avec handi "grand" et mendi "montagne", mais il reste à expliquer la présence du - $a$ final de handi qui doit être encore un suff. aga "lieu" contracté. Dans ce cas, on ne comprend plus handiaga "lieu de grand" (?) et il faut envisager autre chose, sans doute andu -aga "lieu de souches", soit "montagne du lieu des souches".

Harchuria $481 \mathrm{~m}$, comm. d'Ispoure.

Écrit Harchury chez P. Raymond. Étymologie sans problème, de harri "pierre, roche" et xuri "blanc", soit "la roche blanche".

Hardoia $100 \mathrm{~m}$, comm. d'Ustaritz.

Nom composé de harri "pierre, roche" et de doi "ensemble".

Harguibel $935 \mathrm{~m}$, comm. des Aldudes.

Nom composé de harri "pierre, roche" et gibel "arrière".

Haritchartéko ordokia $900 \mathrm{~m}$, comm. de Sainte-Engrâce.

Nom composé de haritz "chêne" et de arte "entre" + ordoki "plateau", soit "le plateau entre les chênes".

Haritzé $500 \mathrm{~m}$, comm. de Saint-Just-Ibarre.

Nom signifiant "chêne", avec $-e$ final postiche.

Harpidey $433 \mathrm{~m}$, comm. de Jaxu.

Nom composé de harri "pierre, roche" + bide "chemin" + egi "crête". Soit "crête du chemin de roche".

Harribeltzéta $918 \mathrm{~m}$, comm. de Licq-Athérey.

Écrit Harribelcéta chez P. Raymond. Nom composé de harri "pierre, roche" + beltz "noir" + eta "lieu", soit "lieu des roches noires".

Harrigorri $1117 \mathrm{~m}$, comm. de Banca.

Nom composé de harri "pierre, roche" et de gorri "rouge".

Harrigorri gagné $1358 \mathrm{~m}$, connm. de Sainte-Engrâce.

Comme le précédent, "hauteur de la pierre rouge".

Harizpuru $282 \mathrm{~m}$, comm. de Caro.

Nom composé de haritz "chêne" et de buru "extrémité".

Hasgagnia $1003 \mathrm{~m}$, comm. d'Aussurucq.

Nom composé de has "roche" (pour haitz) et de gain "hauteur", soit simplement "sommet rocheux".

Heguieder $896 \mathrm{~m}$, comm. d'Estérençuby et de Lécumberry.

Nom composé de hegi "crête" et de eder "beau", soit la "belle crête".

Heguiharitze $500 \mathrm{~m}$, comm. de Juxue.

Nom composé de hegi "crête, bord" et de haritz "chêne".

Héguilaburra $396 \mathrm{~m}$, comm. de Saint-Martin-d'Arrossa.

Nom sans problème, de hegi "crête" et labur "courte".

Heguilla chorrotchia $1680 \mathrm{~m}$, comm. de Larrau.

Nom composé de hegi "crête, bord" et de zorrotz "aigu". Reste à expliquer le segment -la-. Il pourrait s'agir de gibela "l'arrière" ou de bela "le noir". 
Heguilusia $170 \mathrm{~m}$ comm. d'Uhart-Mixe.

Nom composé de hegi "crête" et de luze "longue".

Heylé gagné $1689 \mathrm{~m}$, comm. de Sainte-Engrâce.

Nom composé de hegi "bord, crête" et de -le, segment que l'on observe dans d'autres noms en Soule comme etxele, etc. Il s'agit sans doute d'une forme courte de leku "lieu".

Hido 518 m, comm. d'Aussurucq.

Ce nom est une déformation probable de ihi-doi "lieu, ensemble de joncs".

Hocha handia $571 \mathrm{~m}$, comm. d'Iholdy et de Lantabat.

On trouve en contrebas à $431 \mathrm{~m}$, Hocha chipia "le petit Hocha", correspondant à ce "grand Hocha". Ce Hocha est certainement hotz-a "le froid", c'est-àdire le "mont froid".

Hoharreko kaskoa 295 m, comm. de Saint-Étienne-de-Baïgorry.

On peut envisager ici un nom formé de uharre, soit "le sommet du torrent"?

Hostatéguy 1142 m, comm. d'Arnéguy.

On ne peut séparer ce nom de celui du village d'Hosta, de même étymologie. Il faut analyser cet oronyme à partir de hotz + ta "lieu de froid", soit "crête du lieu froid".

Hourdespary $1006 \mathrm{~m}$, comm. de Larrau.

Oronyme peut-être composé de urd- "plateau" + aitz "pierre, roche" + pe "sous" + iri "domaine".

Huberleta $720 \mathrm{~m}$, comm. de Saint-Étienne-de-Baïgorry.

Nom très déformé, sans doute de hur-bel-eta "lieu d'eau noire".

Ibantelli 698 m, comm. de Sare.

Écrit Ibantelly chez P. Raymond. Sans doute de ibar "vallée" + - $t_{-}+$+ eli "troupeau".

Ichtauz $1024 \mathrm{~m}$, comm. de Banca.

Nom composé de aitz "pierre, roche" + -t- + l'oronyme auz. Soit "montagne de rochers".

Ichterbegui 1027 m, comm. d'Urepel.

Écrit Izterbéguy chez P. Raymond. A première vue, ce nom signifie "ennemi". Ce pourrait être l'appellation populaire d'une montagne dangereuse ou menaçante. Mais on peut aussi y voir iztar, ezter "gorge" + egi "crête", plus conforme à l'oronymie.

Idoikobizkarra $425 \mathrm{~m}$, comm. de Sare.

Nom composé de ihi-doi "lieu de joncs" + ko + bizkar "dos, crête", soit "crête du lieu des joncs".

Iguzki $844 \mathrm{~m}$, comm. d'Itxassou.

Nom sans problème pour ce pic, de iguzki "soleil".

Ihatia $1271 \mathrm{~m}$, comm. d'Aussurucq.

Ce sommet est le plus élevé de la forêt des Arbailles. Il pourrait bien s'agir d'une déformation souletine de Iratia "le lieu des fougères". 
Ihisu $464 \mathrm{~m}$, comm. de Bidarray.

Nom composé de ihi "jonc" et du suff. -su, soit lieu "où les joncs abondent".

Ihizgorri $555 \mathrm{~m}$, comm. de Jaxu.

Cette hauteur, qui se nomme aussi Nabahandi "grande plaine à proximité des montagnes", est formé de ihiz "jonchaie" et gorri "rouge, sec".

Inchouriste $717 \mathrm{~m}$, comm. de Haux.

Nom déformé sans doute, à partir de gain "haut" et xuri "blanc" + suff. -tz(e) $>$ zte.

Iparla 1044 m, comm. de Saint-Étienne-de-Baïgorry.

Ecrit Ipharla chez P. Raymond. Nom composé de ipar "nord" et d'un vieux suffixe -la observé dans plusieurs oronymes (cf. Larla, Arla) et qui ne peut pas être ola "cabane" comme dans Ibarla/Ibarrola. L'altitude du pic d'Iparla exclut ola "cabane". On n'y trouve que des bordes. Peut-être s'agit-il de alha "pâturage" (?).

Iramendy $866 \mathrm{~m}$, comm. d'Estérençuby et de Saint-Michel.

Nom composé de ira "fougère" et de mendi "montagne".

Iraukotuturu $1050 \mathrm{~m}$, comm. de Lécumberry.

Écrit Iraucotuturu chez P. Raymond. Nom composé de ira "fougère" $+u+k o$ + tuturru "sommet".

Iriapizta $379 \mathrm{~m}$, comm. d'Ossès.

Mauvaise graphie de l'IGN. On dit sur place Irapiztia ou Ilapiztia, de ira "fougère" ou ilhar "bruyère". Le -a final est long, donc c'est un ancien -aga "lieu". Le second élément piztia demeure obscur, peut-être un ancien albiztiaga "lieu de fourrage".

Ispéguy $672 \mathrm{~m}$, comm. de Saint-Étienne-de-Baïgorry.

Le nom de ce col est composé de iz "pierre, roche" (var. de aitz) + pe "sous, en bas" + egi "crête, bord". C'est-à-dire la "crête au pied de la roche", laquelle roche doit être sans doute l'Auza tout proche. On a souvent écrit à tort que ce nom signifiait "vue sur la mer", prétendu composé de iz "mer" et begi "œil, vue"!

Issarbe $1565 \mathrm{~m}$, comm. de Sainte-Engrâce.

Écrit Isarbe chez P. Raymond. Une forêt porte le même nom. On peut envisager ici soit izar "étoile", soit izal "sapin" suivi de be "sous, en bas", mais dans ce dernier cas on s'explique mal le $-r$ final de izar. On notera que izar semble avoir servi de vieil oronyme avec le sens de "hauteur".

Itchachéguy $1161 \mathrm{~m}$, comm. de Saint-Michel.

Écrit Itchashéguy chez P. Raymond. Nom composé de itsas "genêt" et de egi "crête".

Izeyto $1464 \mathrm{~m}$, comm. de Sainte-Engrâce.

Un bois porte le même nom. On peut envisager izei "sapin", mais pourquoi alors ce -to diminutif "petit sapin"? En fait le bois porte le nom de la montagne qui n'est pas boisée, sauf à considérer que c'est l'oronyme qui tiendrait son nom du bois, ce qui n'est pas impossible. Il est peut-être préférable d'analyser ce nom en $i z$ "pierre, roche" + egi "crête" contracté en $e i+$ diminutif $t o$. On aurait donc dans ce cas "la petite crête rocheuse" (?). Mais izei "sapin" ne peut pas être exclu à priori. 
Jara $812 \mathrm{~m}$, comm. d'Irouléguy.

Écrit Jarra chez P. Raymond. La graphie de Raymond est fantaisiste et francophone, car on a ici de toute évidence une var. jara du terme xara "bois, taillis".

Jaura 1069 m, comm. de Sainte-Engrâce.

Il existe un bois et une maison du même nom. On peut difficilement faire appel à jaur-qui est en basque une forme de jaun "seigneur" qui n'apparaît que dans les mots composés (jauregi "château", etc.). Le dictionnaire de Dauzat, Deslandes, Rostaing signale un oronyme Jaur dont le sens demeure obscur. Il y a aussi un toponyme Jaur en Dordogne et une rivière du même nom dans l'Hérault.

Kalbarioa 275 m, comm. d'Urrugne.

Petite hauteur qui, comme son nom l'indique, signifie simplement "le calvaire".

Kokoitché 389 m, comm. de Chéraute.

C'est un nom anthropomorphique dérivé de kokots "menton" qui prend ici le même sens que kokor "sommet".

Koskoroy 705 m, comm. de Saint-Étienne-de-Bä̈gorry.

Nom dérivé de koskor var. possible de kokor, konkor "sommet, bosse" (?) + oi. Mais koskor, koxkor signifie aussi "grain, morceau", ce qui nous donnerait peutêtre "qui a tendance à se détacher en morceaux" si l'on considère -oi comme un suff. de tendance. Pour la terminaison -oi on pourra aussi comparer avec Chamboroy (voir ce nom à sa place alphabétique).

Kurutchégagna $1486 \mathrm{~m}$, comm. de Larrau.

Nom sans problème, de kurutze "croix ou croisement" et gain "hauteur".

Laxun-ordoquy $188 \mathrm{~m}$, comm. de Chéraute.

Nom composé de lats "cours d'eau" et de un "lieu" + ordoki "plateau" Soit "plateau du Iieu de cours d'eau".

Laina $869 \mathrm{~m}$, comm. de Bidarray.

Il s'agit sans doute ici d'une forme réduite, peut-être un ancien larregaina "sur la lande, hauteur de la lande".

Lakhoura 1877 m, comm. de Sainte-Engrâce.

Le nom de ce pic, écrit Lacourre chez P. Raymond, est peut-être d'origine romane (gasconne) plutôt que basque.

Lapitsague $406 \mathrm{~m}$, comm. de Viodos-Abense-de-Bas.

Nom composé de lapitz "roche marneuse" et du suff, -aga "lieu".

La Rhune $900 \mathrm{~m}$, comm. de Sare, Ascain et Urrugne.

Ce nom célèbre est en fait une horrible francisation d'un nom basque. Le $\mathrm{La}$ initial a été pris pour l'article défini féminin français. Il convient évidemment de rétablir la vraie forme Larhun, Larrun qui signifie "lieu de lande".

Laurigna $1278 \mathrm{~m}$, comm. de Banca.

Nom obscur qui rappelle Lauribar, peut-être dérivé de laur "quatre" (?). La finale pourrait être gaina "la hauteur".

Lecharra 992 m, comm. d'Alçay-Alçabéhéty-Sunharette.

Nom dérivé sans doute de leizar "frêne" dont la sifflante passe à $x$ en Soule. 
Léchoukohéguia $600 \mathrm{~m}$, comm. de Saint-Martin-d'Arrossa.

Nom obscur composé de lexu ou lexo + ko + hegi "crête". Peut-être leze "gouffre" ou lexuin "fossé" (?).

Leiciaguemendi $335 \mathrm{~m}$, comm d'Ainharp.

Nom composé de leize "goufre" et de -aga "lieu" + mendi "montagne".

Leizar athéka 1409 m, comm. d'Arnéguy,

Écrit Leïçarathéca chez P. Raymond. Nom composé de leizar "frêne" et de atheka "col".

Leizealdia $426 \mathrm{~m}$, comm. de Juxue.

Nom composé de leize "goufre" et de alde "côte".

Lerdatzé 330 m, comm. d'Armendarits et de Saint-Esteben.

Nom composé de ler, leher "pin" + -d- + aitz "pierre, roche". Soit "la roche des pins".

Liétamendy $272 \mathrm{~m}$, comm. d'Ahaxe-Alciette-Bascassan.

Nom composé semble-t-il de ligi "boue" + eta "lieu" + mendi "hauteur".

Lindus $1220 \mathrm{~m}$, comm de Banca.

Écrit Lindux chez P. Raymond, D'une racine romane *lind- "limite". Les terrains ont toujours été très disputés entre haute et basse Navarre.

Lomendi $540 \mathrm{~m}$, comm. d'Etchebar.

Nom qui semble composé de lohi "boue" (?) et mendi "hauteur".

Losco 1108 m, comm. de Sainte-Engrâce.

Il s'agit très certainement du souletin losco "bourbier, marais".

Lumaberdé $226 \mathrm{~m}$, comm. de Biriatou.

Nom composé sans doute de l'espagnol loma "dos" et de berde "vert".

Machela $550 \mathrm{~m}$, comm. de Sauguis-Saint-Etienne.

Nom signifiant "joue", c'est-à-dire "flanc, versant de montagne".

Malgorry $250 \mathrm{~m}$, comm. de Bidarray.

Oronyme composé de la vieille racine pré-indoeuropéenne *mal "montagne" et de gorri "rouge".

Mandale $573 \mathrm{~m}$, comm. de Biriatou et d'Urrugne.

Ce nom semble composé de mando "mulet" et de ale, alha "pâturage".

Matalon $439 \mathrm{~m}$, comm. de Mauléon.

On a affaire sans doute ici à un nom gascon. La base est peut-être mata "hallier, taillis" connu en vieux castillan (espagnol moderne matorral "id.", béarnais matarâ "hallier").

Mataria $812 \mathrm{~m}$, comm. de Lasse.

Écrit Mattaria chez P. Raymond. Peut-être de la base mata "hallier, taillis". La finale pourrait être harri "pierre, roche".

Matchecortia $508 \mathrm{~m}$, comm. de Gotein-Libarrenx.

Nom composé sans doute de mahats "raisin, vigne" et de gorte "aire, cour, enclos". 
Mehalçu $648 \mathrm{~m}$, comm. de Juxue et de Pagolle.

Écrit Méhalçu chez P. Raymond. Nom dérivé de mehe "étroit, défilé, col". Le second élément pourrait être haltzu "aulnaie".

Mendibel $1411 \mathrm{~m}$, comm. de Béhorléguy.

Écrit Mendibels chez P. Raymond. Nom composé de mendi "montagne" et bel "noire".

Mendibile $457 \mathrm{~m}$, comm. d'Tholdy.

Nom composé de mendi "montagne" et de bil "ronde".

Mendichaharra 497 m, comm. de Saint-Étienne-de-Baïgorry.

Nom composé sans doute de mendi "montagne" et de xara "taillis" plutôt que xahar "vieux".

Mendicoi $201 \mathrm{~m}$, comm. d'Aroue-Ithorots-Olhaiby.

Il s'agit peut-être ici de mendiko "petite montagne", sinon mendi-goi "haute montagne", ce qui s'accorde mal avec la faible altitude, bien qu'en basque, mendi puisse désigner des hauteurs très modestes.

Mendicourré $345 \mathrm{~m}$, comm. de Mendionde.

Nom composé de mendi "montagne" et de gorri "rouge, sec".

Mendikao $391 \mathrm{~m}$ comm. d'Hélette.

Il faut sans doute lire ce nom mendikoa "la petite montagne".

Menditipikobizkarra $872 \mathrm{~m}$, comm. d'Itxassou.

Nom composé de mendi-tipi "petite montagne" et de bizkar "crête".

Mispiatségui $305 \mathrm{~m}$, comm. de Chéraute.

Nom composé de mizpiratze "néflier" et de egi "lieu, bord, crête". Soit "crête des néfliers".

Mitserki $800 \mathrm{~m}$, comm. de Larrau.

Nom obscur.

Mocorréta $680 \mathrm{~m}$, comm. de Lécumberry.

Nom composé de mokor "motte" et du suff. -eta "lieu".

Mokoa 358 m, comm. d'Urrugne.

Nom sans problème, de moko "pointe, nez, bec".

Mondarrain $749 \mathrm{~m}$, comm. d'Itxassou.

Cet oronyme constitue un des pièges les plus redoutables que puisse trouver un chercheur non averti. Sous son apparence basque ou à la rigueur mixte, avec mond qui pourrait être roman et arrain qui pourrait être basque, se cache un ancien Montferrand complètement déformé et basquisé. Il apparaît dans l'enquête de 1249 sur la guerre de Thibaud $I^{\text {er }}$ de Navarre en Labourd : castrum de Monte Ferrandi.

Monhoa $558 \mathrm{~m}$, comm. d'Urepel.

Il s'agit simplement de monho "colline, hauteur".

Mougaretta $259 \mathrm{~m}$, comm. de Lohitzun-Oyhercq.

Nom composé de mugarri "borne" et du suff. locatif -eta. 
Mulhédoy $1596 \mathrm{~m}$, comm. de Larrau.

Nom composé d'un premier élément obscur et du suff. collectif-doi. Ce premier élément est peut-être une déformation de ambulo "asphodèle", soit une évolution ambula-doi $>$ amuledo $i>$ muledoi.

Mulugagne $443 \mathrm{~m}$, comm. de Juxue.

Nom composé peut-être de ambulo "asphodèle" déformé + gain "hauteur".

Murrubeltza $1656 \mathrm{~m}$, comm. de Sainte-Engrâce.

Nom composé de murru "sommet" et de beltz "noir".

Murruoin $603 \mathrm{~m}$, comm. des Aldudes.

Nom composé de murru "sommet" et de oin "pied".

Murrutchégagné $1035 \mathrm{~m}$, comm. de Sainte-Engrâce.

Nom composé de murru "sommet" et peut-être de etxe "maison" + gain "hauteur" ou du suff. -tze.

Murrutchipia $1632 \mathrm{~m}$, comm. de Sainte-Engrâce.

Nom composé de murru "sommet" et de txipi "petit".

Muruche $1010 \mathrm{~m}$, comm. de Lacarry-Arhan-Charritte-de-Haut.

Nom composé de murru "sommet" et du suff. collectif -tze.

Nabarlatz $524 \mathrm{~m}$, comm, de Sare.

Nom composé de nabar "de couleur variéc" ou même "Navarre" et de latz "rude, abrupt" ou lats "cours d'eau".

Negoucharo $1002 \mathrm{~m}$, comm. d'Arnéguy.

Nom composé de negu "hiver" ou "neige" et de xaro/zaro "pâture".

Négumendi l $307 \mathrm{~m}$, comm. de Larrau.

Écrit Négumendy chez P. Raymond. Nom composé de negu "hiver" ou "neige", peut-être avec le sens de "pâture d'hiver" et de mendi "montagne".

Néthé $764 \mathrm{~m}$, comm. de Hosta et d'Ibarrolle.

Nom obscur. Il s'agit sans doute d'un vieil oronyme pré-indoeuropéen. Hors du Pays basque, on trouve le pic de Nédé $1648 \mathrm{~m}$, (Ariège).

Occabe $1466 \mathrm{~m}$, comm. de Lécumberry.

Écrit Occabé chez P. Raymond. Nom à mettre en relation avec le toponyme Occos (Okoze) et les Montes de Oca du Pays basque sud, réinterprétés populairement par "monts de l'oie". On pourrait y voir une vieille racine oronymique préindoeuropéenne apparentée à *jok-/juk-, elle-même forme affaiblie de l'oronyme pré-indoeuropéen kuk "hauteur". On la retrouve dans le toponyme Jokoberro à Saint-Étienne-de-Baïgorry et peut-être dans Juxue. On aurait donc ici une forme réduite ok-suivie de - be "sous, en bas". Soit "en bas de la hauteur".

Occostey $371 \mathrm{~m}$, comm. de Saint-Étienne-de-Baïgorry.

Nom inséparable bien sûr du village d'Occos. Il faut donc traduire par "crête d'Occos", avec egi "crête" réduit à ei et - $t$ - de liaison.

Oianalékomendia $318 \mathrm{~m}$, comm. d'Ossès.

Nom composé de oihan "forêt" + ale + ko + mendi "montagne". Soit "la montagne de" 
Olalargy $747 \mathrm{~m}$, comm. de Licq-Athérey.

Nom composé de ola "cabane" et de larre "lande" + egi "crête, bord".

Oneaga $381 \mathrm{~m}$, comm. d'Urrugne.

Nom composé sans doute de one "colline" et de aga "lieu".

Orgambidesca $1445 \mathrm{~m}$, comm. de Lécumberry et Larrau.

Nom composé de orga "charrette" + bide "chemin" + diminutif -ska, soit "le petit chemin des charrettes".

Orgamendy $639 \mathrm{~m}$, comm. de Gamarthe, Larceveau et Ibarrolle.

Nom composé de orga "charrette" et de mendi "montagne".

Orhy $2017 \mathrm{~m}$, comm. de Larrau.

Le nom de ce pic est obscur. Peut-être faudrait-il y voir orri "feuille" ou orre "genévrier" (?).

Orisson $1064 \mathrm{~m}$, comm. de Saint-Michel.

Cet oronyme avait la forme Lorizun au XVI e siècle : Sancta Mariae Magdalenae de Lorizun. Mais cela ne prouve pas que le $l$ - initial de cette forme soit vraiment étymologique. Il peut malgré tout s'agir de l'article français agglutiné. En 1686 on trouve Arisson: Sancta Maria Magdalena d'Arisson. C'est le nom d'un ancien prieuré et aussi celui d'un bois. On pourrait envisager d'expliquer ce nom à l'aide de orre "genévrier", suffixz -tz ou aitz "pierre" + un "lieu".

Otchogorrigagna $1923 \mathrm{~m}$, comm. de Larrau.

Écrit Otxogorry chez P. Raymond. Nom sans problème, composé de otso "loup" + gorri "rouge ou sec, dénudé" + gain "hauteur", sans doute s'agit-il d'une allusion à l'aspect du lieu ou à quelque mythe ou récit.

Otchogorritehipia $1794 \mathrm{~m}$, comm. de Larrau.

Nom composé de otso-gorri "loup rouge" et de txipi "petit". C'est "le petit Otxogorri" par opposition au précédent plus élevé.

Othamonho $762 \mathrm{~m}$, comm. d'Hosta et de Saint-Just-Ibarre.

Nom composé de othe "ajonc" et monho "colline, hauteur", soit "hauteur des ajoncs".

Othegagne $513 \mathrm{~m}$, comm. de Juxue.

Écrit Othégagne chez P. Raymond. Nom composé de othe "ajonc" et de gain "hauteur". Soit "hauteur des ajoncs".

Otsamunho $901 \mathrm{~m}$, comm. des Aldudes et de Banca.

Nom composé de otso "loup" et de munho "colline, hauteur".

Oyhergain $347 \mathrm{~m}$, comm. de Lohitzun-Oyhercq.

Le nom de cette hauteur est certainement en rapport avec celui du village tout proche d'Oyhercq. C'est donc la "hauteur d'Oyhercq".

Oylarandoy $930 \mathrm{~m}$, comm. de Saint-Étienne-de-Bägorry.

Écrit Oillarandoy chez P. Raymond. Nom composé de oillaran "prunellier" (le terme goillaran "prunellier" est encore connu du dialecte roncalais) et du suff. doi. Soit "ensemble de prunelliers". 
Pagaburu $543 \mathrm{~m}$, comm. de Lantabat.

Écrit Phagabure chez P. Raymond. Nom composé de pago "hêtre" et de buru "sommet, extrémité". Soit "extrémité des hêtres".

Patarbeltcha $1200 \mathrm{~m}$, comm. de Lécumberry.

Nom composé de patar "forte pente" et de beltz "noir".

Patarramonho $881 \mathrm{~m}$, comm. d'Urepel.

Nom composé de patar "forte pente" et de monho "colline, hauteur". Le terme patar est un vieux terme substratique pyrénéen pré-indoeuropéen que l'on trouve aussi en gascon (gascon petarrè "penchant abrupt"). Cf. le pic Pétar(d) dans le département des Hautes-Pyrénées $(2548 \mathrm{~m}$ ). Le mot supporterait assez bien la comparaison avec le finnois pättärä "colline". On le trouve aussi en dravidien (patar "colline"). Il n"a strictement rien à voir avec le français pente.

Patzé $270 \mathrm{~m}$, comm. d'Isturits.

Ce nom représente sans doute une contraction de pago "hêtre" suivi du suff. -tze.

Péliusegagne $1594 \mathrm{~m}$, comm. de Larrau.

Nom obscur, sans doute dérivé d'une vieille racine pré-indoeuropéenne *pelvar. de * pal/bal bien attestée en oronymie. On peut comparer avec la Pointe Pelouse en Haute-Savoie (2474 m). On est donc dans ce cas en présence d'une tautologie "hauteur de la montagne".

Penzezabala $493 \mathrm{~m}$, comm. de Juxue.

Nom composé de pentze "pré" et de zabal "plat, large". Soit "le plat ou plateau des prés".

Pista $1779 \mathrm{~m}$, comm. de Larrau.

Nom d'origine romane probablement. Cf. gascon pista "piste".

Saiberri $505 \mathrm{~m}$, comm. de Sare.

Écrit Sayberry chez P. Raymond. Ce nom pourrait être une contraction de sabai "grange" suivi de berri "nouvelle", car on voit mal ce que voudrait dire "nouveau vautour".

Sakia Gagnékoa $1374 \mathrm{~m}$, comm. de Larrau.

Nom composé de saki "entaille, crevasse" et de gain "hauteur".

Sakiko Achourterrigagna $1528 \mathrm{~m}$, comm. de Larrau.

Écrit Achourtéry chez P. Raymond. Nom composé de saki "entaille, crevasse" pour le premier élément, et de axuri "agneau" + (h) arri "pierre, roche" + gain "hauteur" pour le second. Il y a en Soule une maison Axurbidegi "crête du chemin des agneaux". La montagne ci-dessus se nomme donc "la hauteur du rocher des agneaux de la crevasse".

Salhagagne $1053 \mathrm{~m}$, comm. de Larrau.

Nom composé de salha "maison noble, salle" et de gain "hauteur". Soit "salle d'en haut" ou "hauteur de la salle".

Saltéburia $970 \mathrm{~m}$, comm. de Larrau.

Nom sans doute composé de zaltu "saut, forêt" (au sens du saltus latin) et de buru "sommet, extrémité" avec fermeture en $i$ devant l'article. Le é de Salté demeure cependant inattendu. 
Saminékohéguy $533 \mathrm{~m}$, comm. de

Le basque samin "goût acide" est peu vraisemblable ici. Il faut chercher une explication à partir de sagar-min "plantation de pommiers" ou à la rigueur à partir de lamina "lutin" qui se serait altéré par cacographie. Soit "crête de la plantation de pommiers".

Sare-Sare $806 \mathrm{~m}$, comm. de Mendive.

Nom formé en apparence du redoublement de sare. En réalité il doit y avoir deux mots distincts à l'origine. D'une part sans doute sare "filet" et d'autre part xara "taillis, petit bois". Le "filet" faisant sans doute allusion à une palombière.

Sarimendi $1484 \mathrm{~m}$, comm. de Larrau.

Nom composé de sarri "broussailles, taillis" et de mendi "montagne".

Sarleguigagna $636 \mathrm{~m}$, comm. de Haux.

Nom composé sans doute de sari "fourré" ou xara "taillis" + $l$ - de liaison + egi "crête" + gain "hauteur". Soit "la hauteur de la crête des pommiers".

Saukalde 638 m, comm. d'Estérençuby. côté".

Nom composé de sabuka "sureau" contracté en sauka et de alde "région ou

Seineguy $593 \mathrm{~m}$, comm. d'Ahaxe-Alciette-Bascassan.

Il existe un curieux nom identique mais avec la graphie Saint Héguy à proxi-mité, sur la commune voisine de Lécumberry. Il s'agit d'un faux hagionyme (faux. nom de saint). On analysera plutôt ce nom comme zeina "signe de croix" + egi "crête" ou à la rigueur à partir de itzain "bouvier" qui aurait perdu son premier élément.

Selata punta $1050 \mathrm{~m}$, comm. d'Aussurucq.

Nom composé de zelai "plateau" et du suff. -eta "lieu". Soit "pointe du lieu. de plateau".

Sen Julian $476 \mathrm{~m}$, comm. de Jaxu.

Nom roman passé en basque. Donc mont "Saint-Julien".

Sihigue $1193 \mathrm{~m}$, comm. d'Aussurucq.

Il s'agit du même nom que le village de Cihigue. De zihi "gland, chêne" ou "jonc" et aga "lieu".

Sombiague $861 \mathrm{~m}$, comm. de Licq-Athérey.

Nom sans doute déformé. Peut-être à partir de halzu "aulnes" + be "en bas" + aga "lieu". Cf. par ex. le toponyme Sumberraute qui est une forme réduite de haltzu-m-berro-eta "lieu du taillis des aulnes". Le $m$ est une anticipation de la labiale $b$.

Sorhogain $820 \mathrm{~m}$, comm. d'Urepel.

Nom composé de sorho "prairie, champ" et de gain "hauteur".

Soylandotchipi $551 \mathrm{~m}$, comm. d'Hélette.

Écrit Soylando chez P. Raymond. Ce nom pourrait être une cacographie de ihitz-oilaran-doi "lieu des prunelliers de la jonchaie" + txipi "petit". Sinon on peut aussi envisager zohi "motte de terre" comme premier élément. 
Subisia $568 \mathrm{~m}$, comm. d'Urrugne.

Écrit Subissia chez P. Raymond. Ce nom semble comporter zubi "pont" comme premier élément, mais c'est peut-être trompeur. On peut aussi envisager un zuhi-tz "lieu d'arbres, de chênes" mal écrit.

Suhalmendi $301 \mathrm{~m}$, comm. de Sare.

II s'agit probablement ici d'un composé de zuhar "orme" + mendi "mont, hauteur". Soit "mont des ormes".

Tharta punta $1521 \mathrm{~m}$, comm. de Larrau.

Écrit Tarta chez P. Raymond. Nom composé de tartha "chêne vert" (forme souletine du plus général arte, arta "id.") et de punta "pointe". Soit "pointe des chênes verts".

Tourouna $220 \mathrm{~m}$, comm. de Garris.

Nom roman, cf. gascon turon "touron", ici sous une forme basquisée.

Toutoulia 983 m, comm. de Saint-Étienne-de-Baïgorry.

Nom d'origine expressive signifiant simplement "sommet" (tuturu, etc.).

Trempetta 802 m, comm. de Saint-Étienne-de-Baïgorry.

Nom roman (gascon) sans doute, peut-être simplement "trempette" dans une région où il pleut beaucoup.

Trépoyko cascoa $165 \mathrm{~m}$, comm. de Méharin.

Nom probablement gascon, sans doute "le sommet des trois puys" (gascon tre (s)pośy).

Tuc de Biscarrague $91 \mathrm{~m}$, comm. d'Urcuit et d'Urt.

Nom composé du gascon tuc "hauteur, sommet" et du basque bizkar "id." + aga "lieu". Soit le "tuc du lieu de crêtes". Comme mendi "montagne", le basque bizkar "dos, crête, etc." peut s'appliquer à des petits sommets de faible altitude (ici $91 \mathrm{~m}$ ). Cf. par ex. Biscarosse dans les Landes.

Tuc du Bouc $51 \mathrm{~m}$, comm. d'Urt.

Comme son nom l'indique! Forme gasconne francisée.

Tuquet $225 \mathrm{~m}$, comm. d'Etcharry.

Nom gascon au diminutif. Soit "la petite hauteur, le petit tuc".

Ulhunaguerré punta $1084 \mathrm{~m}$, comm. de Sainte-Engrâce.

Nom composé de ulhun "sombre", var. de ilhun "id." + agerre "en vue". Soit "pointe de l'endroit sombre (un bois?) en vue". Le terme ilhun "sombre" s'expliquerait bien par il-un "lieu sombre" (lieu de lune, de ténèbre) qui s'opposerait ainsi à egun "jour" qui serait eg-un "lieu de soleil".

Unkhugnérri $1218 \mathrm{~m}$, comm. de Larrau.

Nom composé du souletin unkhü "souche" + un "lieu" + (h)arri "pierre, roche". Soit "roche du lieu des souches".

Urchamendy $176 \mathrm{~m}$, comm. d'Arbouet-Sussaute.

Nom composé de urso "colombe, palombe" et mendi "hauteur".

Urchilo 500 m, comm. de Saint-Martin-d'Arrossa.

Nom composé de ur "eau" et de xilo "trou". 
Urculu 1419 m, comm. de Saint-Michel.

Écrit Urculo chez P. Raymond. On admet généralement que ce nom de montagne où domine la "tour" romaine bien connue est issu de urkulu "cercle", du latin circulum "id.". On ne peut toutefois pas exclure d'autres hypothèses, comme par ex. un nom dérivé de urki "bouleau".

Urdanarré $1240 \mathrm{~m}$, comm. de Saint-Michel.

Nom composé de l'oronyme urd- "plateau" ou de urda-gain "haut plateau" contracté en urdan suivi de -arre qui peut être soit une var. de arri "pierre, roche", soit aurre "avant, devant".

Urdanasburu $1233 \mathrm{~m}$, comm. de Saint-Michel.

Nom composé de urda-gain "haut plateau" + aitz "pierre, roche" + buru "extrémité". Soit "extrémité rocheuse du haut plateau".

Urdiakoharria $996 \mathrm{~m}$, comm. de Saint-Étienne-de-Baïgorry.

Nom composé probablement de urd "plateau" + egi "crête, bord, lieu" + aga "lieu" + ko + harria. Soit "la roche du lieu de crête du plateau".

Uritzaray $200 \mathrm{~m}$, comm. de Saint-Martin-d'Arberoue.

Nom composé de urritz "coudrier" et de garai "hauteur" contracté.

Urritzgaray $273 \mathrm{~m}$, comm. de Saint-Martin-d'Arberoue. driers",

Nom composé de urritz "coudrier" et de garai "haut", soit "hauteur des cou-

Urrizpilota 935 m, comm. de Saint-Étienne-de-Baïgorry.

Nom composé sans doute de urritz "coudrier" + bil "tas, forme ronde, hauteur" + eta "lieu". La graphie correcte serait donc Urritzpileta.

Ursoa $455 \mathrm{~m}$, comm. d'Ossès.

Nom composé en principe de urso "palombe" et aga "lieu". Cependant on ne peut exclure totalement un rapport avec le nom même du village d'Ossès, ancien Orz-aiz dont le premier élément représente le viel oronyme *urz-, var. de urd"plateau", attesté au X $\mathrm{X}^{\mathrm{c}}$ siècle (Urz-ahaiz).

Ursuya $678 \mathrm{~m}$, comm. de Macaye.

Nom composé de ur "eau" et du suff. -tsu. Soit "lieu où l'eau abonde".

Uthurri Hotchépunta $1708 \mathrm{~m}$, comm. de Larrau.

Nom composé de uthurri "source"(var. souletine de ithurri) + hotz "froide" + punta "pointe". Soit "pointe de la source froide".

Utzigagna $1618 \mathrm{~m}$, comm. de Sainte-Engrâce.

Nom composé peut-être de $u z i$ "partage" + gain "hauteur".

Xoldokocana $486 \mathrm{~m}$, comm. de Biriatou.

Écrit Choldocogagna chez P. Raymond. Nom dont le premier élément est obscur, peut-être composé de saldo "troupeau" + ko + gain "hauteur". Soit "la hauteur des troupeaux" (?).

Zabozé 1178 m, comm. de Saint-Just-Ibarre.

Écrit Cabocé chez P. Raymond. Nom peu clair, sans doute constitué d'une forme réduite de zabarotz (attesté comme nom de maison médiévale) ou zabalotz.

Zalgazareteitzaleta $797 \mathrm{~m}$, comm, de Lécumberry. 
Nom composé de deux parties bien distinctes : zalga-zahar-eta d'une part et itzal-eta de l'autre. Soit "lieu d'ombre (itzal-eta) du lieu de la vieille ivraie". C'est bien l'un des noms les plus longs de la toponymie basque!

Zarkindegui $860 \mathrm{~m}$, comm. des Aldudes.

Écrit Sarguindéguy chez P. Raymond. Curieux nom peu clair. Ce pourrait être sarekin-degi "maison du fabricant de filets" ou une mauvaise graphie de hargin "maçon, tailleur de pierres" (?). Il y a en effet une maison Sarkindéa en contrebas de cette hauteur.

Zazpigagn $1765 \mathrm{~m}$, comm. de Larrau.

Nom composé de zazpi "sept" et de gain "hauteur". Soit "les sept sommets".

Zègnhaguia $1240 \mathrm{~m}$, comm. de Sainte-Engrâce.

Nom signifiant "lieu de signe". C'est-à-dire sans doute "lieu où il faut se signer" (faire le signe de croix) ou bien à la rigueur "lieu du signal".

Zeztokokaskoa 495 m, comm. de Saint-Martin-d'Arrossa.

Nom sans doute composé de zuhaitz-to "petit arbre" très fortement contracté + ko + kasko "sommet". Soit "sommet, hauteur du petit arbre".

Zohuta $575 \mathrm{~m}$, comm. d'Urepel.

Nom qui semble être un soro-eta "lieu de champs" contracté.

Zurrusta gaïna $700 \mathrm{~m}$, comm. de Lasse.

Nom composé de zurrusta "chute d'eau" et de gain "hauteur". Soit "la hauteur de la chute d'eau". 